\title{
Comparação entre métodos de estimativa da Evapotranspiração de Referência (ETo) na região de Petrolina-PE
}

\author{
Comparasion of methods the Evaporation Reference (ETo) in the region of Petrolina-PE
}

Hérika Cavalcante Dantas da Silva*1, Madson Tavares Silva², Mislayne Mayana Moura Araújo Silva ${ }^{3}$, Carlos Alberto Nascimento da Rocha Junior ${ }^{4}$

1,3,4 Graduandos de Ciências e Tecnologia, UFRN.

${ }^{2}$ Mestre, Professor Substituto DEC/CT/UFRN.

\begin{abstract}
Resumo
O trabalho objetivou estimar a evapotranspiração de referência (ETo) diária para a região de Petrolina-PE, através de dados da estação agrometeorológica de Bebedouro, da unidade de pesquisa da EMBRAPA-SEMIÁRIDO. Para tanto, foram utilizados dados meteorológicos referentes ao ano de 2010 na estimativa dos métodos de PenmamMonteith-FAO (PM), de Linacre, da Radiação e do Tanque Classe A. Adotou-se o método de PM como parâmetro para avaliar a eficiência dos demais e o indice (c), de Camargo e Sentelhas, para avaliar o desempenho dos mesmos. A ETo pelo método de PM chegou a apresentar valores médios diários de 3 a $8 \mathrm{~mm}$ dia-1. De acordo com os resultados, foi observado que os métodos da Radiação e de Linacre obtiveram resultados satisfatórios, alcançando desempenho ótimo, ou seja, valores acima de 0,85 para c no período que compreende os meses de setembro a janeiro. Porém, com relação ao erro relativo a PM, o método de Linacre se mostrou bastante eficiente para a área analisada, já que apresentou um valor de $0.76 \%$, enquanto o método do Tanque Classe A mostrou os piores resultados, com $12 \%$. Já o método da Radiação, apesar do bom coeficiente de desempenho, apresentou subestima a PM, com um erro de $-13 \%$.
\end{abstract}

Palavras-chave: Semiárido, evaporação do tanque, manejo de irrigação.

\begin{abstract}
The study was aimed to estimate the reference evapotranspiration (ETo) daily for the region of Petrolina-PE, using data from the weather station Trough, inserted in the research unit of EMBRAPA-SEMIARID (CPATSA). Were used meteorological data for the year 2010 in the estimation methods of Penmam-Monteith-FAO (PM), of Linacre, Radiation and Class A pan. We adopted the method of PM as a parameter to evaluate the efficiency of other methods and the index (c) of Camargo and Sentelhas to evaluate their performance. For the method of PM was observed that in general the ETo values show great variability throughout the year in the semiarid region of the state of Pernambuco, reaching present daily average values between 3,0 and 8,0 $\mathrm{mm}$ day-1. According to the results it was observed that the methods of radiation and Linacre obtained satisfactory results, as the performance criterion, values above 0,85 for $\mathrm{c}$ in the months of September to january. However, with respect to error on the $P M$, the Linacre method was very efficient for the study area, as there was a value $0,76 \%$, while the method of the class A pan method showed the worst results, with an error around $12 \%$. Have the method of radiation, despite the good performance coefficient showed underestimate the PM method, obtaining an error of $-13 \%$.
\end{abstract}

Keywords: Semiarid, pan evaporation, irrigation management.

*herikacavalcante@yahoo.com

Recebido: 13/03/2014 Aceito: 13/03/2014 


\section{Introdução}

$\mathrm{O}$ conhecimento das necessidades hídricas das culturas é de grande importância para o estudo do manejo da água de irrigação. A evapotranspiração é uma das principais variáveis do ciclo hidrológico. Segundo Sediyama (1998), a taxa de evapotranspiração refere-se à transferência de água para a atmosfera, da superfície do solo e das plantas pela combinação da evaporação com a transpiração.

Para se alcançar economia hídrica na agricultura é oportuno se obter uma irrigação mais próxima do ideal e, para tanto, necessita-se de estudos que levem em consideração principalmente os aspectos meteorológicos da região, mas também, se fazer estimativas mais confiáveis para o manejo dessa técnica, em cujo contexto a estimativa adequada da evapotranspiração de referência (ETo) é de suma importância (MENDONÇA \& DANTAS, 2010). A ETo é, segundo Pozzebon et al. (2003), a água evaporada do solo e transpirada pelas plantas em uma superfície coberta por vegetação específica rasteira, uniformemente distribuída, em fase de crescimento ativo, em solo com perfeita condutibilidade hídrica e mantido sempre úmido, próximo à capacidade de campo, sendo assim, de grande importância para o dimensionamento e manejo da água de irrigação.

Dentre os vários métodos utilizados para a estimativa da evapotranspiração de referência, o método desenvolvido por Penman, e posteriorrmente complementado por Monteith, é o considerado como método padrão pela Organização das Nações Unidas para a Agricultura e Alimentação (FAO), por ser mais confiável, sendo comprovado pelo estudo de diversos autores, como Allen (1986, 1998) e Sediyama (1996). Porém o método de Penmam-Monteith (PM) é complexo e depende de muitas variáveis, logo, há a necessidade de se encontrar um método mais simples que se aproxime do padrão adotado pela FAO.

Por isso este trabalho objetiva comparar o método de PM com outros três - o de Linacre, o da Radiação e o do Tanque Classe A - para região de Petrolina-PE, que possui grande potencial agroindustrial, pois devido ao clima seco e à irrigação, tornou-se a maior exportadora de frutas e o segundo maior centro vinícola do Brasil. Desse modo, o conhecimento da melhor e mais simples técnica de estimativa da evapotranspiração assume grande importância econômica.

\section{Material e métodos}

Os dados utilizados nesse estudo foram coletados pela estação agrometereológica de Bebedouro, pertencente à unidade de pesquisa da EMBRAPA-SEMIÁRIDO (CPATSA), localizada em Petrolina-PE, cujas coordenadas geográficas são $09^{\circ} 09^{\prime \prime} \mathrm{S}$, de latitude e $40^{\circ} 22^{\prime \prime} \mathrm{W}$, de longitude.
Foram utilizados dados diários de temperaturas máxima, mínima e média; umidade relativa; insolação; radiação; velocidade do vento; evaporação do tanque classe A; e pressão; compreendidos entre janeiro e dezembro de 2010.

O método de Penmam-Monteith-FAO é dado pela Equação (1):

$$
\text { EToPM }=\frac{0,408 \Delta\left(R_{n}-G\right)+\gamma \frac{900}{T_{a}+273} u_{2}\left(e_{s}-e_{a}\right)}{\Delta+\gamma\left(1+0,34 u_{2}\right)}
$$

Em que: ETo = evapotranspiração de referência $(\mathrm{mm} /$ dia), $\mathrm{Rn}=$ Saldo de Radiação (, G = Fluxo de calor no solo (, Ta Temperaura média do ar $\left({ }^{\circ} \mathrm{C}\right)$, u2 = Velocide média diária do vento a $2 \mathrm{~m}$ de altura, es $=$ Pressão de saturação de vapor média diária $(\mathrm{kPa})$, ea $=$ Pressão atual de vapor média diária $(\mathrm{kPa}), \Delta=$ Declividade da curva de pressão de vapor no ponto correspondente a temperatura $\left({ }^{\circ} \mathrm{C}\right), \gamma=$ Consante psicométrica $\left(\mathrm{kPa} /{ }^{\circ} \mathrm{C}\right)$.

O método descrito por Linacre é descrito pela Equação (2):

$$
\text { EToL }=\frac{500 \frac{\mathrm{T}_{\text {med }}+(0,006 \mathrm{H})}{100-(\mathrm{L})}+15\left(\mathrm{~T}_{\text {med }}-\mathrm{T}_{\mathrm{O}}\right)}{\left(80-\mathrm{T}_{\text {med }}\right)}
$$

Em que: = Temperatura média $\left({ }^{\circ} \mathrm{C}\right),=$ Altitude $(\mathrm{m})$, para a localidade do estudo foi adotado $375 \mathrm{~m}$, =Latitude (m), para a localidade do estudo foi adotado $-9,15 \mathrm{~m}$, $=$ Temperatura de Orvalho $\left({ }^{\circ} \mathrm{C}\right)$.

A evapotranspiração de referência (ETo) da região foi calculada através do método da radiação de acordo com a Equação (3):

$$
\mathrm{EToR}=\mathrm{C} * \mathrm{~W} * \mathrm{R}_{\mathrm{S}}
$$

Em que: $\mathrm{C}=$ coeficiente angular de regressão $\mathrm{ETo} \times \mathrm{W}$ $\mathrm{Q}$, determinado em função da umidade relativa média do ar e da velocidade média do vento a $2 \mathrm{~m}$ de altura, $\mathrm{W}=$ fator que representa a parte fracional da radiação solar que é utilizada na ETo, para diferentes valores de temperatura e altitude, =radiação solar global medida, cal dia.

Tanque Classe A - método direto que consiste em medir a lâmina d'água evaporada em um tanque padrão e multiplicar seu valor por um coeficiente de segurança, segundo a Equação (4):

$$
\text { EToTCA }=\text { Evap. } * \text { Kt }
$$

Tais cálculos foram realizados com o auxílio de planilhas do software Excel. Para se obter dados diários foi realizada a média dos dados horários da estação agrometeorológica. Dias que não possuíam dados foram descartados para que não afetassem os resultados. 
Foram realizadas análises de regressão para avaliar o desempenho dos modelos, obtendo-se os coeficientes de determinação (R2), coeficientes de correlação (r), erro médio percentual (EMP), índice de Willmott (d) (Willmott et al., 1981) e índice de desempenho (c) (Camargo \& Sentelhas, 1997).

Através do Excel foram plotados gráficos de dispersão, pelos quais é possível extrair os coeficientes de determinação (R2) e de correlação (, que indicam o grau de dispersão dos dados obtidos, o erro aleatório, não considerando o sistemático.

O erro médio e percentual (EMP) é a diferença entre os valores observados nos métodos que se deseja avaliar e os valores do método que se tomou como parâmetro. Ele é representado pela Equação 5:

$$
\operatorname{EMP}(\%)=\frac{1}{N} \sum_{i=1}^{N}\left|\frac{X_{i}-X_{i}^{\prime}}{X_{i}^{\prime}}\right| \times 100 \%
$$

Em que: $X$ representa o valor observado, $X^{\prime}$ representa o valor estimado a partir dos modelos e $\mathrm{N}$ representa o número de valores.

$\mathrm{O}$ índice d quantifica numericamente a exatidão, sendo um coeficiente de concordância (Willmott et al., 1981). Ele é obtido através da Equação (6) a seguir:

$$
\mathrm{d}=1-\frac{\sum_{\mathrm{i}}^{\mathrm{N}}\left(\mathrm{X}-\mathrm{X}^{\prime}\right)^{2}}{\sum_{\mathrm{i}}^{\mathrm{N}}\left(\left|\mathrm{X}^{\prime}-\overline{\mathrm{X}}\right|+|\mathrm{X}-\overline{\mathrm{X}}|\right)^{2}}
$$

Em que: = média dos valores observados.

$\mathrm{O}$ índice de desempenho c é dado pelo produto do coeficiente de correlação (r) e o índice de Willmott (d) (Camargo e Sentelhas, 1997) conforme a Equação (7):

$$
\mathrm{c}=\mathrm{r} * \mathrm{~d}
$$

Tabela 1. Valores de (c) Conforme Camargo \& Sentelhas (1997).

\begin{tabular}{cc}
\hline Valor de c & Desempenho \\
\hline$>0,85$ & Ótimo \\
$0,75-0,85$ & Muito bom \\
$0,66-0,75$ & Bom \\
$0,61-0,65$ & Mediano \\
$0,51-0,60$ & Sofrível \\
$0,41-0,50$ & Mau \\
$\leq 0,40$ & Péssimo \\
\hline
\end{tabular}

\section{Resultados e discussão}

A partir da estimativa da ETo pelos métodos descritos, procurou-se identificar uma equação que melhor se ajustasse ao método de Penman-Monteith FAO e que envolvesse uma quantidade menor de parâmetros nos cálculos, de modo a se obter, de forma mais simples, a estimativa da Evapotranspiração de Referência.

Para a localidade e ano analisados, o mês de março mostrou-se como o mais chuvoso, enquanto que o mais seco foi o de agosto. Os resultados diários de ETo para esses meses encontram-se nas Figuras 1 e 2, respectivamente. Através da Figura (1) é possível verificar que, para o mês mais chuvoso de 2010 (março) na região de Petrolina, os valores de ETo calculados pelo método da Radiação se ajustam consideravelmente aos valores de PM.

Já no mês de agosto (Figura 2), o mais seco, o método de PM tem os valores mais semelhantes aos de Linacre que aos da Radiação. Em ambos os meses, percebemos que o método do tanque classe A possui valores mais elevados que do método padrão adotado.

A comparação dos métodos com o de Penmam torna-se mais eficaz quando se analisa os valores dos coeficientes de determinação (R2) e correlação (r), e dos índices de Willmott (d) e de desempenho (c), que são apresentados nas tabelas 2 (valores mensais) e 3 (média anual).

Na tabela 2: O é ótimo, MB é muito bom, $B$ é bom, $S$ é sofrível e P é péssimo. Essa classificação é realizada com base na tabela 1 .

Através da tabela 2 é possível observar que em 7 meses (setembro a janeiro, março e abril) do ano de 2010, os valores obtidos pelo método da radiação são considerados ótimos pelo índice c de Camargo \& Sentelhas, obtendo uma vantagem sobre o de Linacre, que possui apenas 3 meses (janeiro, julho e dezembro) com classificação ótima. Em contrapartida, no período de maio a agosto os valores da Radiação obtiveram classificação sofrível, enquanto que o de Linacre obteve, para o mesmo período, classificações melhores como Ótimo, Bom e Muito bom.

Analisando a média anual, mostrada na tabela 3, vemos que tanto a ETo calculada pelo método da radiação quanto a ETo calculada pelo método de Linacre possuem classificação Muito bom, com EToRAD obtendo uma leve vantagem, apresentando um valor de c de 0,78, contra 0,77 de Linacre.

Por fim, a tabela 4 permite visualizar o erro médio a cada mês, bem como a média anual. Analisando tais dados é possível perceber que, apesar de bons resultados, o método da radiação subestima o de Penmam, pois obteve erro médio percentual anual negativo (-13\%). Já Linacre obteve um erro inferior a $1 \%$, característica bastante positiva para tal método.

Como já foi possível visualizar inicialmente, através dos gráficos 1 e 2, o método do tanque classe A obteve valores bem mais elevados que os de Penmam, apresentando, portanto, um erro bem mais elevado que os demais $(12 \%)$ e uma classificação péssima pelo índice c. 


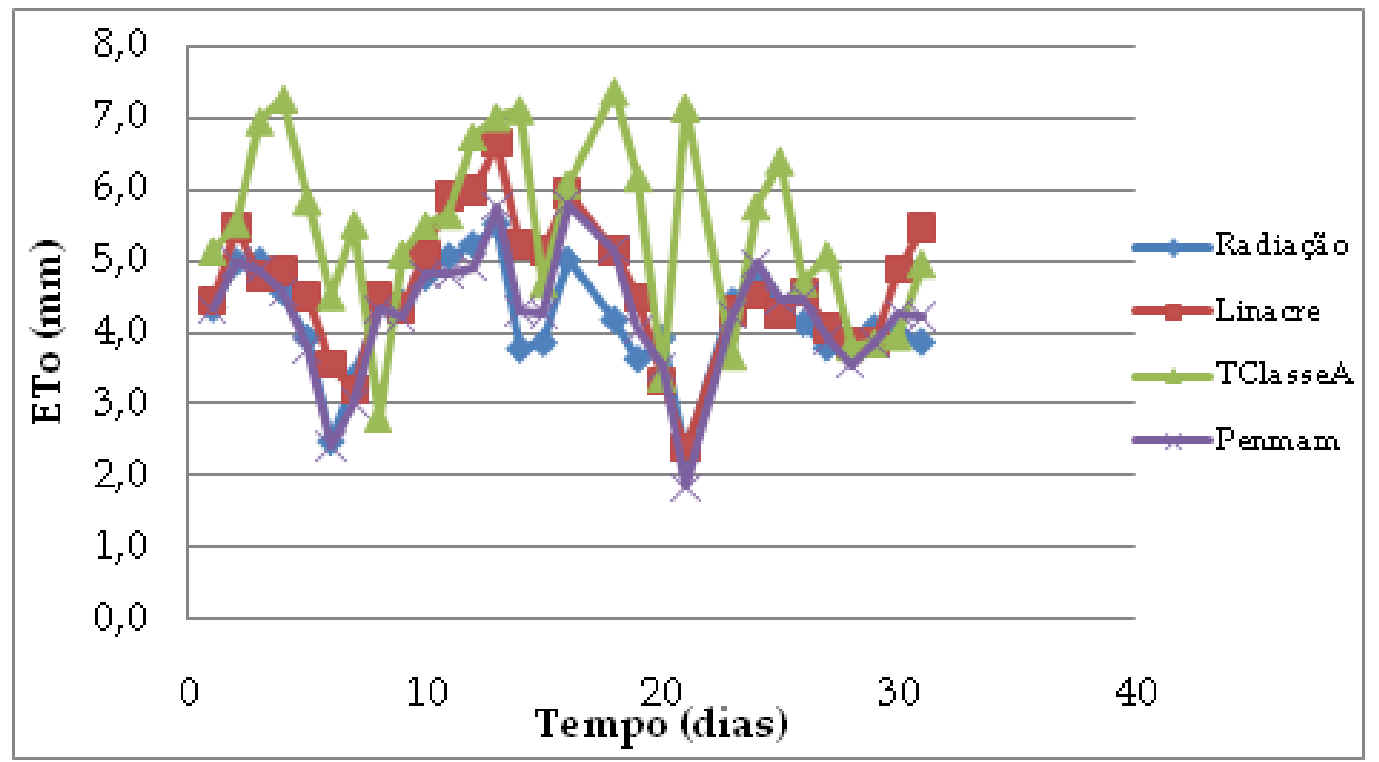

Figura 1. Valores diários de ETo para o mês de Março/2010.

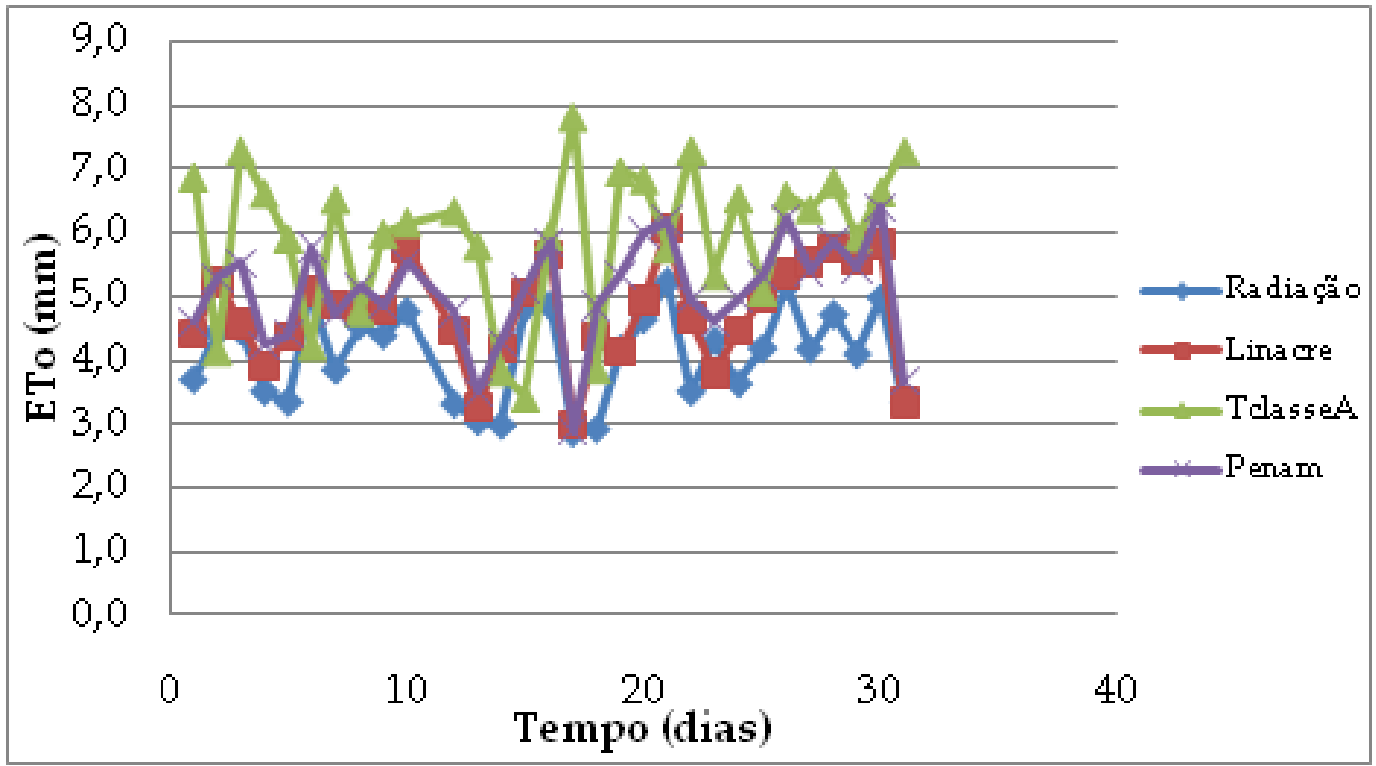

Figura 2. Valores diários de ETo para o mês de Agosto/2010. 
Tabela 2. Valores dos coeficientes e índices para a comparação entre o método de Penmam e demais (Radiação, Linacre e Tanque Classe A).

\begin{tabular}{|c|c|c|c|c|c|c|c|c|c|c|c|c|c|c|c|}
\hline \multirow{2}{*}{ MÊS } & \multicolumn{5}{|c|}{ RADIAÇÃO } & \multicolumn{5}{|c|}{ LINACRE } & \multicolumn{5}{|c|}{ TANQUE CLASSE A } \\
\hline & $\mathbf{r}^{\wedge} \mathbf{2}$ & $\mathbf{r}$ & d & c & Clas & $\mathbf{r}^{\wedge} \mathbf{2}$ & $\mathbf{r}$ & d & c & Clas & $\mathbf{r}^{\wedge} 2$ & $\mathbf{r}$ & d & c & Clas \\
\hline JAN & 0,8 & 0,89 & 1 & 0,89 & $\mathrm{O}$ & 0,87 & 0,93 & 0,96 & 0,9 & $\mathrm{O}$ & 0,57 & 0,75 & 0,68 & 0,51 & $\mathrm{~S}$ \\
\hline FEV & 0,9 & 0,95 & 0,86 & 0,96 & $\mathrm{MB}$ & 0,77 & 0,88 & 0,93 & 0,82 & MB & 0,46 & 0,68 & 0,57 & 0,38 & $\mathrm{P}$ \\
\hline MAR & 0,84 & 0,92 & 0,95 & 0,87 & $\mathrm{O}$ & 0,76 & 0,87 & 0,89 & 0,78 & MB & 0,07 & 0,27 & 0,27 & 0,07 & $\mathrm{P}$ \\
\hline $\mathrm{ABR}$ & 0,93 & 0,96 & 0,98 & 0,94 & $\mathrm{O}$ & 0,69 & 0,83 & 0,9 & 0,76 & MB & 0,16 & 0,4 & 0,44 & 0,18 & $\mathrm{P}$ \\
\hline MAI & 0,17 & 0,41 & 0,54 & 0,58 & $\mathrm{~S}$ & 0,7 & 0,84 & 0,82 & 0,69 & B & 0 & 0,02 & 0,03 & 0 & $\mathrm{P}$ \\
\hline JUN & 0,45 & 0,67 & 0,54 & 0,52 & $\mathrm{~S}$ & 0,54 & 0,74 & 0,8 & 0,59 & $\mathrm{~S}$ & 0,08 & 0,29 & 0,02 & 0,01 & $\mathrm{P}$ \\
\hline JUL & 0,69 & 0,83 & 0,53 & 0,54 & S & 0,8 & 0,89 & 0,94 & 0,85 & $\mathrm{O}$ & 0,03 & 0,16 & 0,24 & 0,04 & $\mathrm{P}$ \\
\hline AGO & 0,72 & 0,85 & 0,64 & 0,54 & $\mathrm{~S}$ & 0,8 & 0,89 & 0,9 & 0,81 & $\mathrm{MB}$ & 0,31 & 0,56 & 0,23 & 0,13 & $\mathrm{P}$ \\
\hline SET & 0,72 & 0,85 & 0,98 & 0,85 & $\mathrm{O}$ & 0,58 & 0,76 & 1 & 0,76 & $\mathrm{MB}$ & 0,42 & 0,65 & 0,99 & 0,64 & $\mathrm{M}$ \\
\hline OUT & 0,78 & 0,88 & 0,99 & 0,85 & $\mathrm{O}$ & 0,36 & 0,6 & 1 & 0,8 & $\mathrm{MB}$ & 0,43 & 0,66 & 0,99 & 0,65 & B \\
\hline $\mathrm{NOV}$ & 0,77 & 0,88 & 0,99 & 0,87 & $\mathrm{O}$ & 0,34 & 0,59 & 0,98 & 0,57 & S & 0,05 & 0,22 & 0,99 & 0,21 & $\mathrm{P}$ \\
\hline DEZ & 0,91 & 0,95 & 1 & 0,95 & $\mathrm{O}$ & 0,9 & 0,95 & 0,99 & 0,94 & $\mathrm{O}$ & 0,32 & 0,56 & 0,98 & 0,55 & $\mathrm{~S}$ \\
\hline
\end{tabular}

Tabela 3. Valores médios anuais dos coeficientes e índices de comparação ente Penmam e os demais métodos.

\begin{tabular}{cccccc}
\hline Método & $\mathbf{r}^{\wedge 2}$ & $\mathbf{r}$ & $\mathbf{d}$ & $\mathbf{c}$ & Classificação \\
\hline RADIAÇÃO & 0,72 & 0,84 & 0,83 & 0,78 & MUITO BOM \\
LINACRE & 0,68 & 0,81 & 0,92 & 0,77 & MUITO BOM \\
TANQUE & 0,24 & 0,44 & 0,54 & 0,28 & PÉSSIMO \\
\hline
\end{tabular}


Tabela 4. Valores mensais do Erro Médio Percentual.

\begin{tabular}{cccc}
\hline \multicolumn{4}{c}{ Erro Média Percentual (EMP)\% } \\
Mês & Rad e PM & Linacre e PM & TA E PM \\
\hline Janeiro & $-5,04$ & 3,76 & 21,27 \\
Fevereiro & $-16,05$ & 2,65 & 20,69 \\
Março & $-0,64$ & 9,93 & 34,26 \\
Abril & 0,04 & 7,9 & 23,62 \\
Maio & $-9,05$ & 8,14 & 11,85 \\
Junho & $-25,82$ & 6,63 & 0,38 \\
Julho & $-21,29$ & $-1,48$ & $-2,76$ \\
Agosto & $-24,13$ & $-7,09$ & 11,06 \\
Setembro & $-30,07$ & $-8,13$ & 8,02 \\
Outubro & $-15,68$ & 3,36 & 10,01 \\
Novembro & $-15,35$ & 3,16 & 18,26 \\
Dezembro & 5,49 & $-19,68$ & $-11,8$ \\
MÉDIA & $\mathbf{- 1 3 , 1 3}$ & $\mathbf{0 , 7 6}$ & $\mathbf{1 2 , 0 7}$ \\
\hline
\end{tabular}

\section{Conclusões}

Dos métodos estudados, o da Radiação e Linacre apresentaram desempenho "muito bom" na estimativa da evapotranspiração de referência em qualquer época do ano, podendo ser recomendados para o uso nos projetos de irrigação na região, uma vez que seus resultados apresentaram uma boa correlação com o método padrão, Penman-Montheith FAO.

\section{Referências}

ALLEN, R. G. A Penman for all seasons. J. Irriga. and Drain. Eng. v. 112, n. 4, p. 348-368, 1986.

ALLEN, R. G.; PEREIRA, L. S.; RAES, K.; SMITH, M. Crop evapotranspiration (guielins for computing grop water requirements). Rome: FAO, 1998. 300 p. (Irrigation and Drainage Paper, 56).

CAMARGO, A. P.; SENTELHAS, P.C. Avaliação do desempenho de diferentes métodos de estimativa da evapotranspiração potencial no estado de São Paulo, Brasil. Revista Brasileira de Agrometeorologia, Santa Maria, v.5, p.89-97, 1997.
MENDONÇA, Ednaldo A.; DANTAS, Renilson T. Estimativa da Evaporanspiração de Referência no município de Capim - PB. Revista Brasileira de Engenharia Agrícola e Ambiental. v.14, n.2, p.196202, 2010. Campina Grande, PB, UAEA/UFCG.

POZEBON, E. J.; Cunha, P.; CAVALCANTI, A. C.; SILVA, L. M. C. Procedimentos para pedidos de outorga de direito de uso da água para irrigação. In: Workshop sobre Água, Agricultura e Meio Ambiente no Estado de São Paulo, 2003. Anais... Jaguariúna: Embrapa Meio Ambiente, 2003.

SEDIYAMA, G. C. Evapotranspiração: necessidade de água para os cultivos. Brasilia: ABAES, 1996. 167p.

SEDIYAMA, G. C. Evapotranspiração: necessidades de água para as plantas cultivada. Brasília, DF: ABEAS, 1998, $181 \mathrm{p}$.

WILLMOTT, C.J. On the validation of models. Physical Geography, v.2, p.184-194, 1981. 